\title{
Reseña del libro: Qualitative Studies in Quality of Life: Methodology and Practice Tonon, Graciela (Ed.) (2015)
}

Heilderberg, Dordretch, New York, London, Springer.

María Juliana Laurito ${ }^{1}$

Universidad de Palermo

Material original autorizado para su primera publicación en el Journal de Ciencias Sociales, Revista Académica de la Facultad de Ciencias Sociales de la Universidad de Palermo.

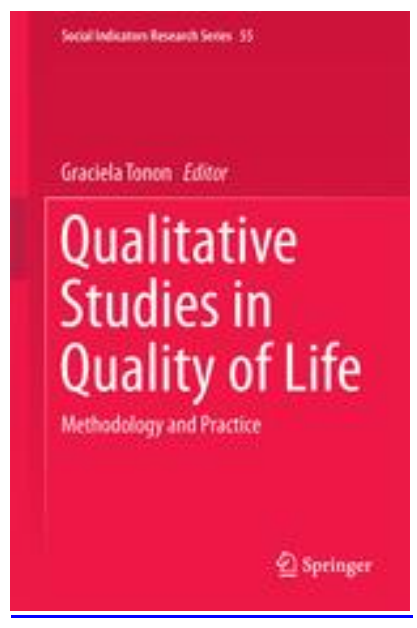

Esta obra es el resultado de un arduo trabajo de la Dra. Graciela Tonon y de un equipo de investigadores: Lía Rodriguez de la Vega, Claudia Mikkelsen, Josefina Di Nucci y Milton Décima (Argentina) Antonio López y María Dilia Mieles Barrera (Colombia) y Lucía Zanabria Ruiz (Perú). Este libro trata sobre la utilización de la metodología cualitativa en los estudios sobre calidad de vida. Ha sido publicado en la prestigiosa Editorial Springer en la colección Social Indicators Research Series.

En los estudios sobre calidad de vida la metodología cualitativa aparece como un desarrollo de vanguardia que permite capturar las palabras, las percepciones y las opiniones de los actores sociales que participan de la investigación, logrando así un mejor acercamiento a la comprensión de la calidad de vida de los sujetos. El texto se encuentra organizado en dos grandes apartados. El primero contiene desarrollos de carácter teórico, mientras que el segundo presenta estudios sobre calidad de vida aplicados a distintos ámbitos, todos en contextos latinoamericanos.

\footnotetext{
${ }^{1}$ mlauri@palermo.edu
} 
La editora es también la autora individual del primero, segundo y cuarto capítulo y en forma compartida del octavo capítulo.

En el primer capítulo Graciela Tonon realiza una valiosa síntesis de la evolución histórica y de los principales hallazgos de los estudios cualitativos en calidad de vida; describiendo las características y especificidades de los métodos cualitativos y mostrando la relevancia de la utilización de la metodología cualitativa en los estudios sobre calidad de vida. En el segundo capítulo la autora aborda el rol del investigador cualitativo, siendo este un tema central para la comprensión y utilización de la metodología cualitativa. Ofrece estrategias y herramientas para llevar a cabo ese papel de manera práctica y concreta y concluye proponiendo diferentes pasos a seguir en el diseño y desarrollo de estrategias metodológicas cualitativas. En el cuarto capítulo la autora aborda la integración metodológica, explicando sus principales particularidades y presentado su utilización en los estudios de calidad de vida. La lectura de este apartado permite entender que la compleja realidad social en la que vivimos requiere ser estudiada a través de una complementación de métodos que posibilite un mejor acercamiento a la noción de calidad de vida.

En el tercer capítulo Lía Rodríguez de la Vega reflexiona e invita al lector a considerar el importante papel del contexto y la cultura en los estudios que tienen como objetivo la comprensión de la calidad de vida. Para ello aborda la noción de cultura y especialmente la relación entre la cultura y la calidad de vida.

En el quinto capítulo Josefina Di Nucci y Claudia Mikkelsen parten de la premisa de que los estudios sobre calidad de vida deberían considerar las apreciaciones y valoraciones que realizan las personas que participan en ellos. Es por ello, que plantean la necesidad de contar con indicadores de carácter cualitativo que faciliten un acercamiento directo a las concepciones de los sujetos.

En el sexto capítulo Milton Décima presenta los hallazgos de un estudio cualitativo que aborda la calidad de vida relacionada con la salud en jóvenes que viven con VIH/SIDA. El autor logra de esta manera trasmitirle al lector una clara noción de que es lo que los jóvenes piensan y sienten sobre esta enfermedad.

En el séptimo capítulo María Dilia Mieles Barrera y Graciela Tonon abordan la calidad de vida de los niños del Caribe desde la metodología cualitativa, favoreciendo la generación de una mirada integral, al tener en cuenta distintas circunstancias de la vida de estos niños.

En el octavo capítulo Lucia Zanabria Ruiz comparte los hallazgos de una investigación correspondiente a su Tesis de Doctorado que llevó a cabo con jóvenes de una comunidad rural y una urbana. El presente estudio tenía como objetivo conocer la satisfacción percibida por los jóvenes con la vida en la comunidad que habitaban, presentando de manera minuciosa las características de los dos grupos de jóvenes participantes. 
En el noveno capítulo Antonio López muestra el desarrollo de un estudio que permite comprender como los jóvenes rurales de la zona andina colombiana construyen sus significados de juventud y la relación que existe entre ellos y el enfoque de juventud asumido por las políticas públicas.

Finalmente en el décimo capítulo Lía Rodríguez de la Vega aborda el alcance de la práctica del Yoga en la calidad de vida en general y en la calidad de vida laboral.

Este libro presenta un excelente relevamiento del estado del arte de la temática, integrando aspectos teóricos y prácticos. El lector puede encontrar aquí los últimos desarrollos e investigaciones del área. Los capítulos que conforman este ejemplar han sido elaborados por autores de diferentes culturas y contextos, aportando su riqueza y diversidad. Para concluir es importante destacar que uno de los aspectos más valiosos de esta obra es el abordaje que realiza de la calidad de vida desde la metodología cualitativa, la cual ha sido escasamente implementada hasta el momento y de esta manera permite abrir un nuevo campo de posibilidades al entendimiento del estudio de la calidad de vida de las personas. 